\title{
Post-processing correction of stray-light in space instruments Application to the 3MI Earth observation instrument
}

- L. Clermont, C. Michel, E. Mazy, Y. Stockman

Presenter: Y. Stockman 
Stray-light requirements are always more stringent

$\rightarrow$ Hardware optimization is not enough, we need post-processing correction 


\section{Outlook}

- Context

- Algorithm

- Data handling

- Measurement methodology

- Conclusions 


\section{MetOp SG - 3MI}

Optical design ( $\pm 57^{\circ}$ FOV)
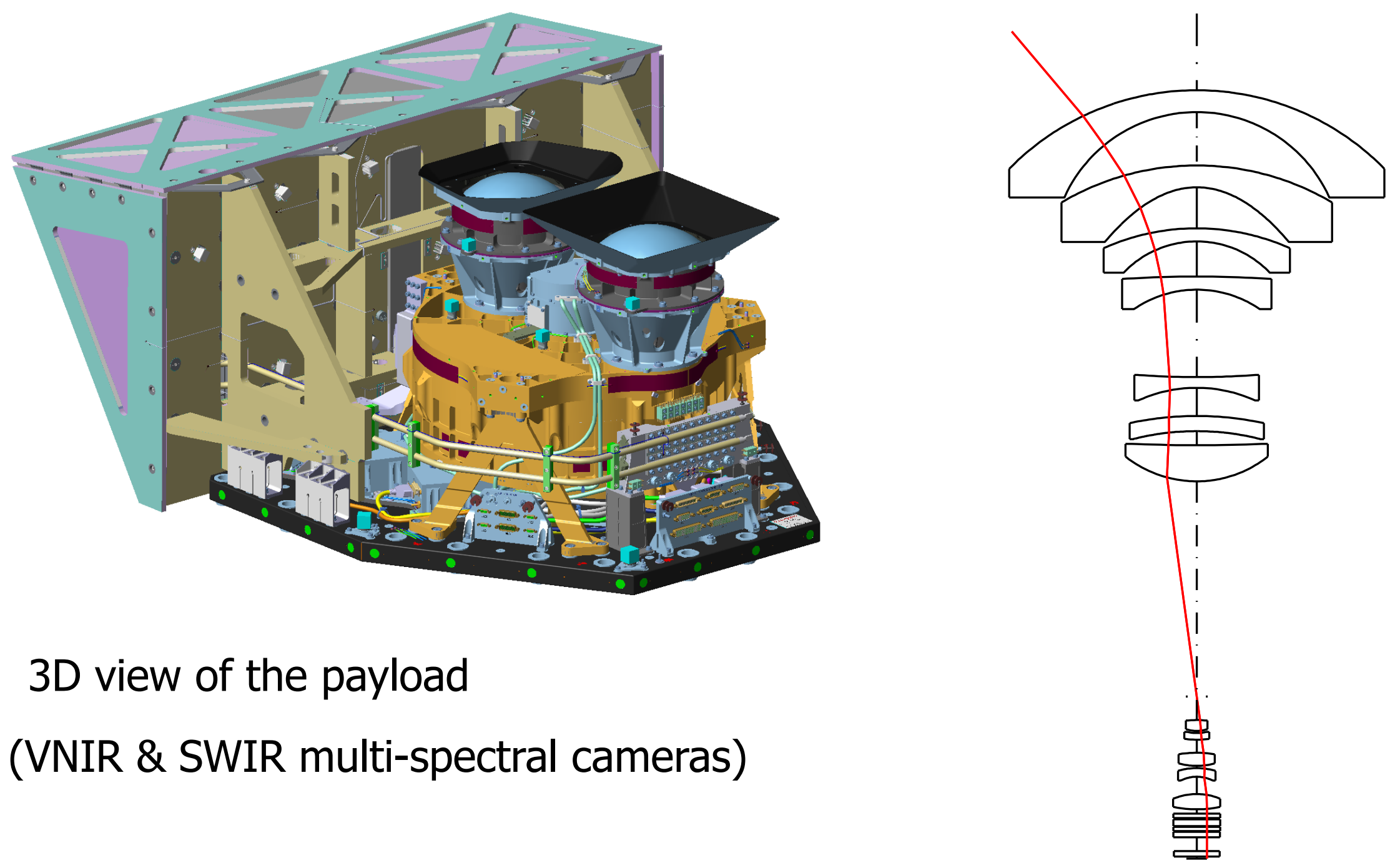

$3 \mathrm{D}$ view of the payload

(VNIR \& SWIR multi-spectral cameras) 
Despite hardware optimization, 3MI has a stray-light up to 2 orders of magnitude larger than what the end users require

Nominal extended scene Associated stray-light
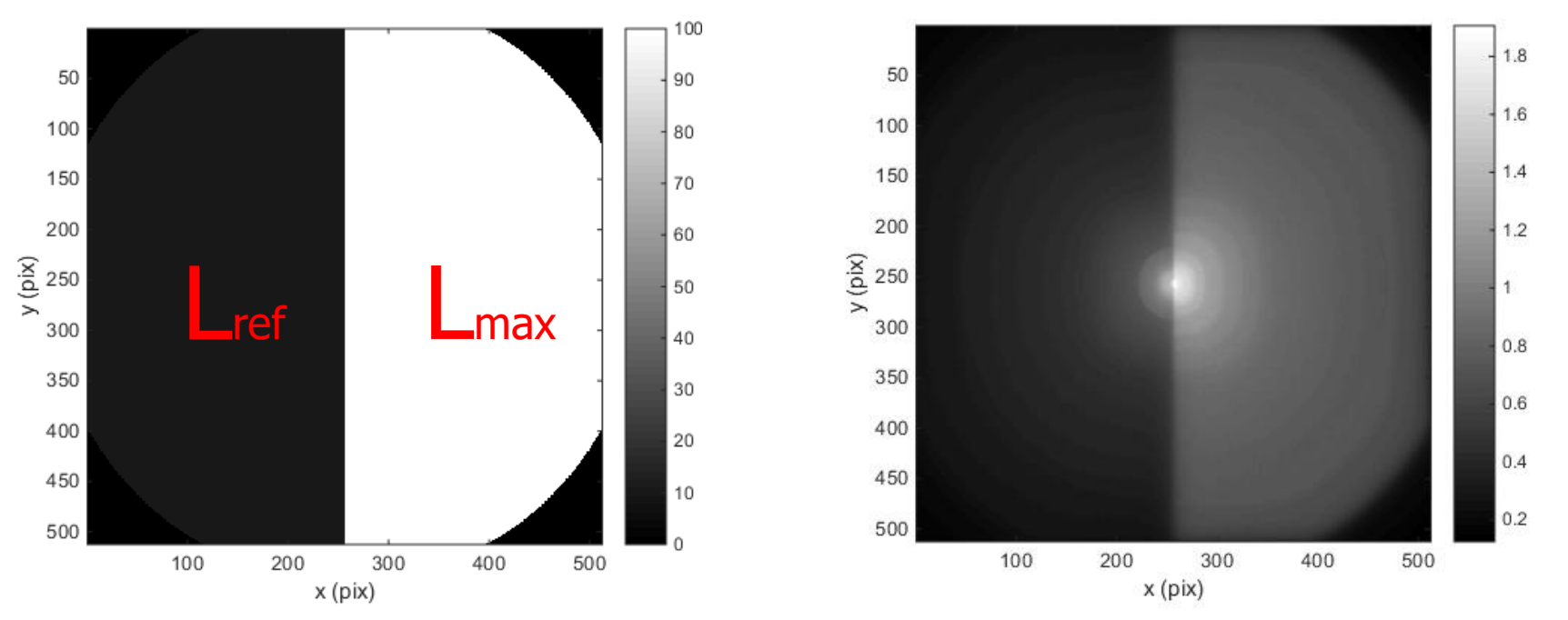

Requirement:

$$
\frac{\left|I_{S}-I_{c o r r}\right|}{I\left(L_{r e f}\right)} \leq 0.17 \% \quad \Rightarrow\left|\Delta I_{S L}\right| \leq 0.17 \% \cdot I\left(L_{r e f}\right)
$$




\section{The algorithm is based on the calibration of SPST maps (Spatial Point Source Transmittance)}

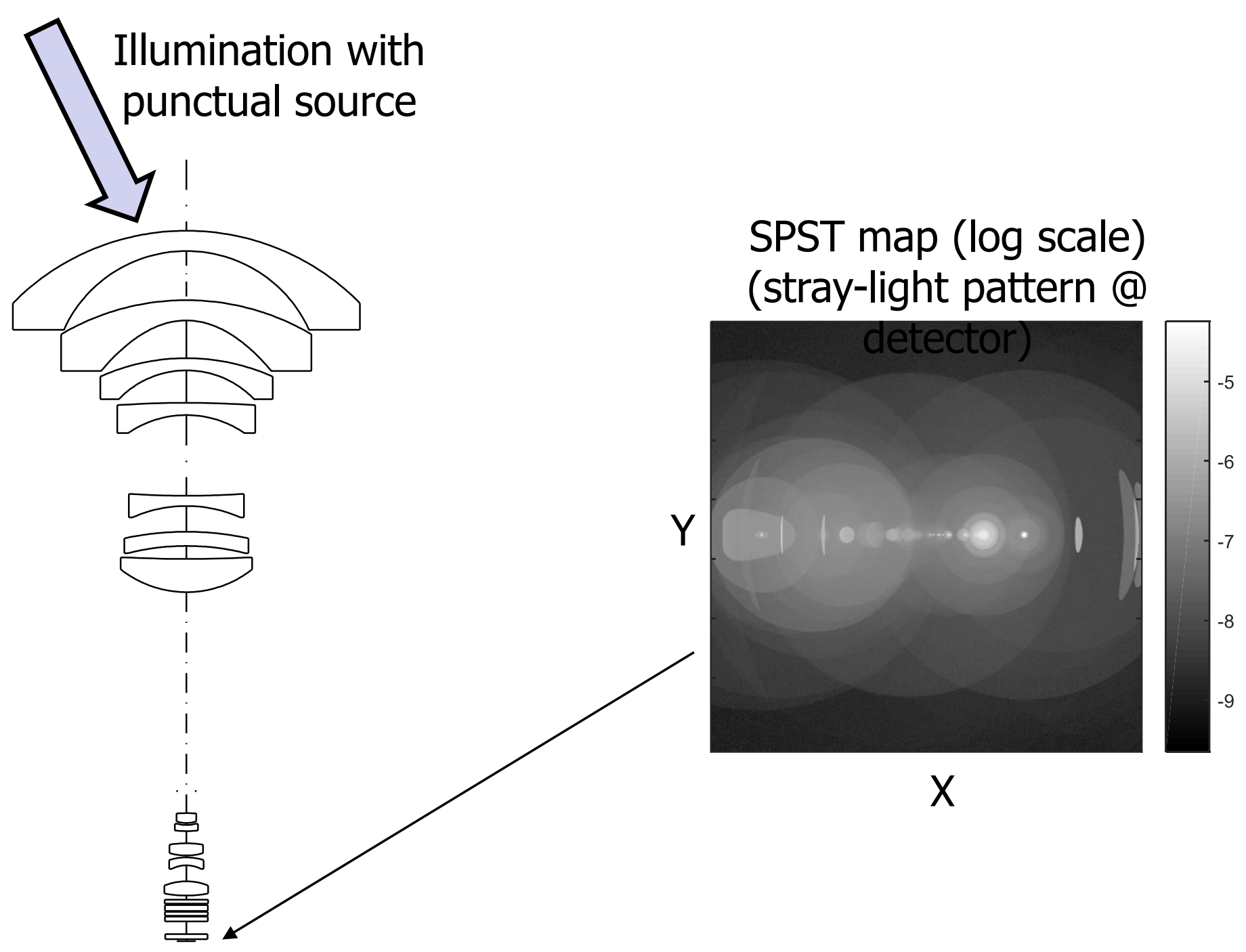




\section{Stray-light is obtained by summing the SPST maps associated to each field, modulated by the nominal signal at the field}

$$
I_{S L}=\sum_{i, j} S P S T_{i, j} \cdot I_{n o m}(i, j)
$$

\section{Matrix} formulation:

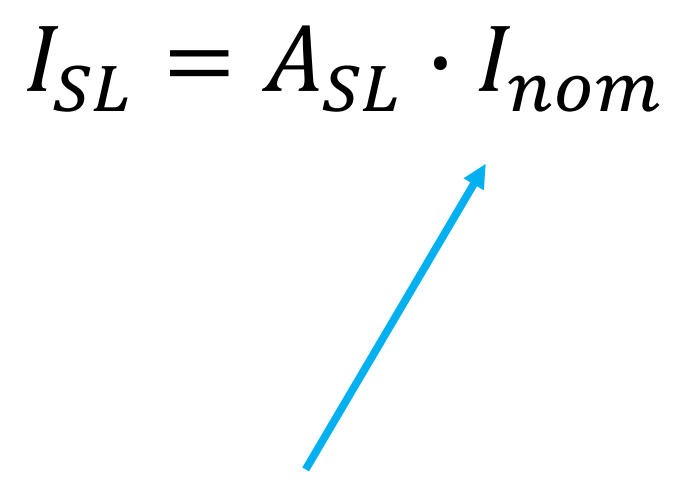

Nominal signal reshaped as a vector

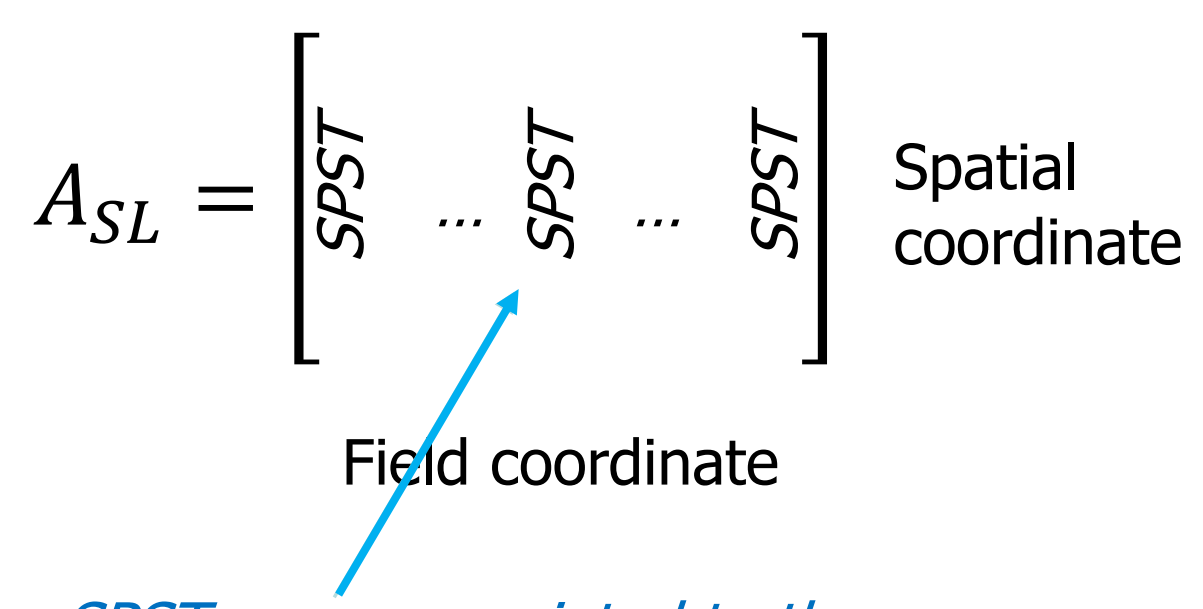

SPST maps associated to the different fields, reshaped as vectors 


\section{Stray-light is estimated by modulating the SPST maps \\ by the measured signal}

Stray-light estimation: $\quad I_{S L, \text { estimated }}=A_{S L} \cdot I_{\text {meas }}$

That map is subtracted from the measured signal:

Corrected image: $\quad I_{\text {corr }}=I_{\text {meas }}-I_{S L \text {,estimated }}$

$\rightarrow$ This process is repeated $\mathbf{n}$ times, replacing $I_{\text {meas }}$ by Icorr at each step 
The residual stray-light level decreases with the number of iterations

\section{Simulation results:}

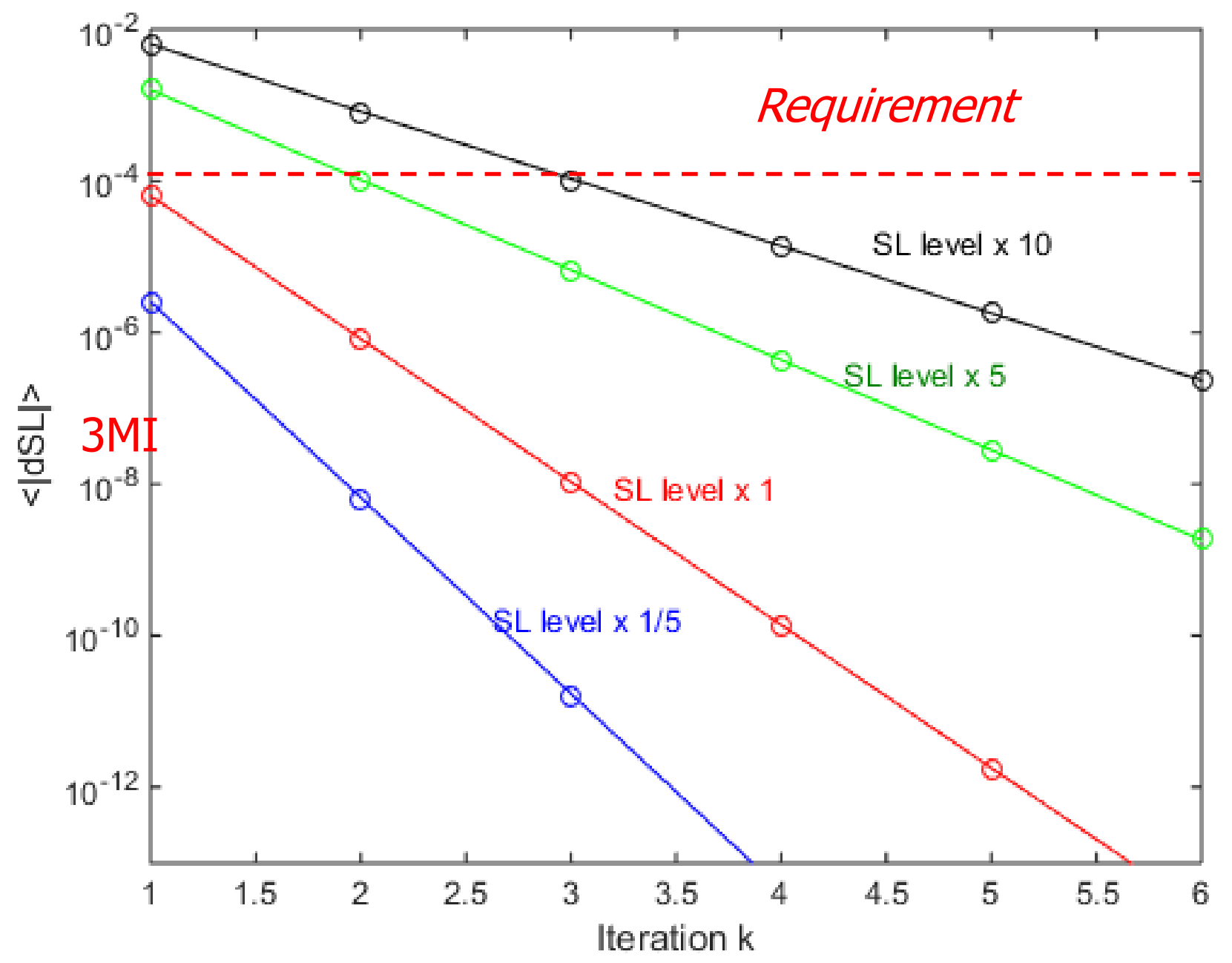

For 3MI, we select 2 iterations 


\section{At full resolution (CCD: $512 \times 512$ pixels), the matrix ASL has $512^{2} \times 512^{2}$ $=67$ billions elements !}

\section{Solutions:}

- Spatial binning: reduction of the resolution of each SPST map

$\rightarrow$ Estimated stray-light in low resolution (not done for 3MI)

- Field binning: averaging SPST maps associated to adjacent fields

$\rightarrow$ Affect modulation of SPST maps (loss of high frequencies)

Implementation:

$$
\begin{aligned}
& I_{S L, \text { estimated }}^{\mathrm{BIN}}=A_{S L}^{B I N} \cdot I_{\text {meas }}^{\text {BIN }} \\
& I_{S L, \text { estimated }}^{\mathrm{BIN}} \rightarrow I_{S L, \text { estimated }} \quad \text { (interpolation) } \\
& I_{\text {corr }}=I_{\text {meas }}-I_{S L, \text { estimated }}
\end{aligned}
$$




\section{Field binning}

\section{Nominal scene}
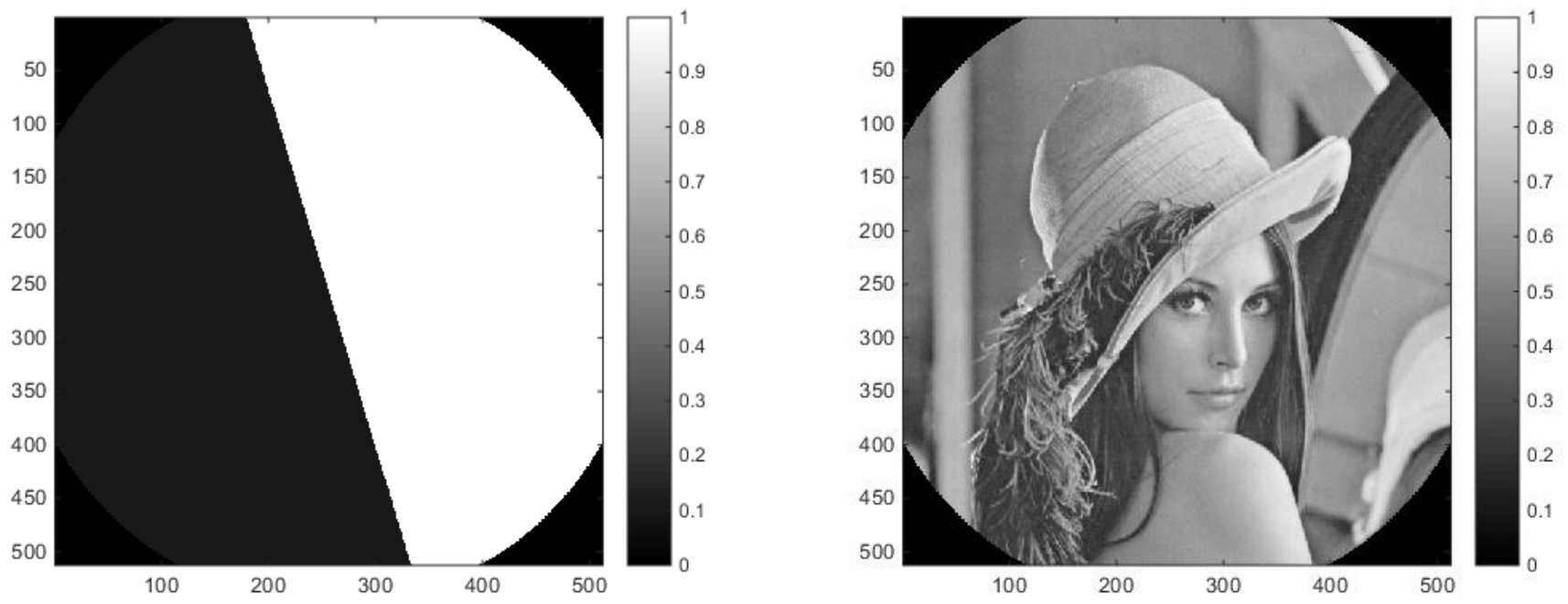

Error on the stray-light correction VS field binning

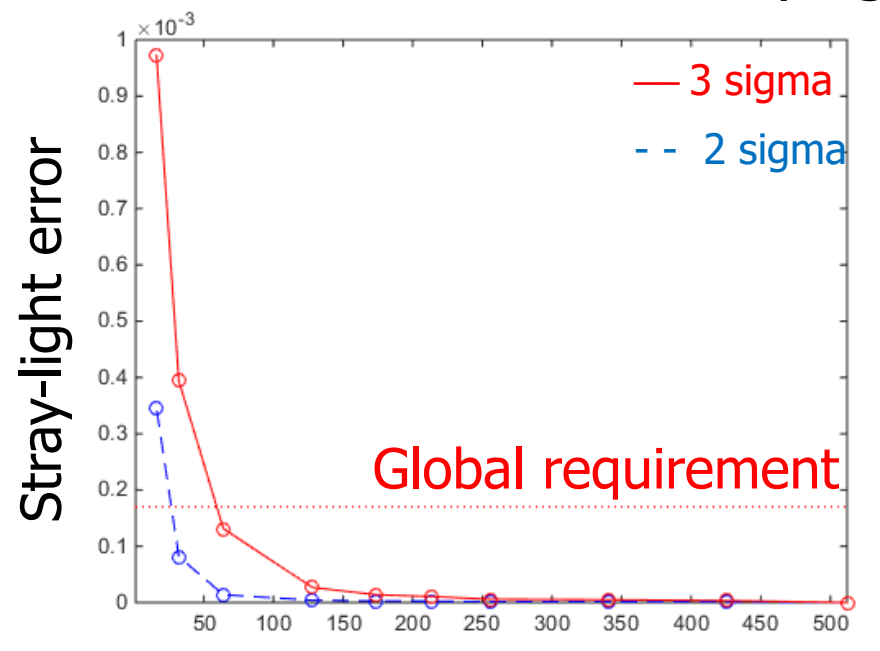

Field binning dimension

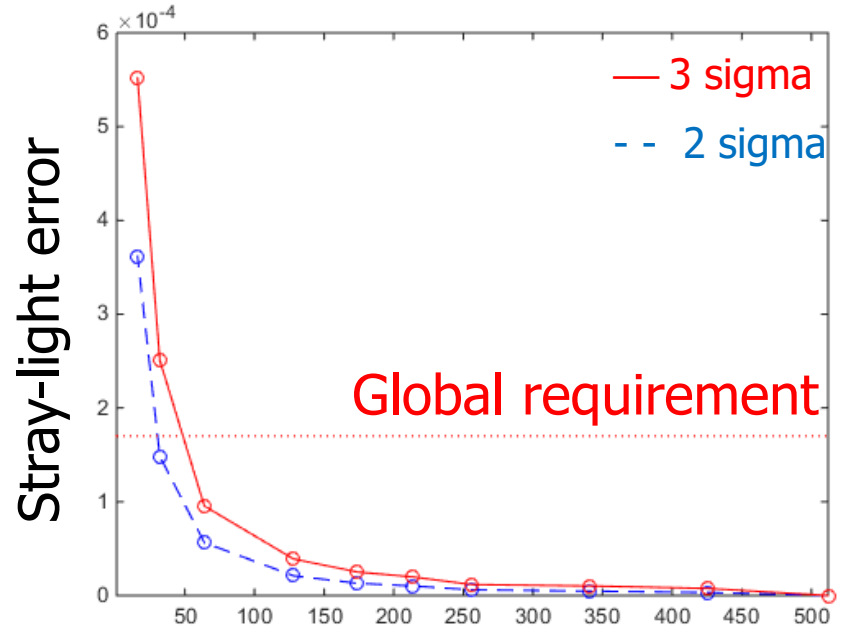

Field binning dimension 


\section{Measurement methodology}

The VNIR detector contains $512 \times 512$ pixels, we can't practically calibrate the SPST for each pixels

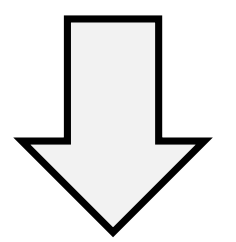

Calibration performed on $27 \times 27$ fields, which makes the SL correction performance very poor

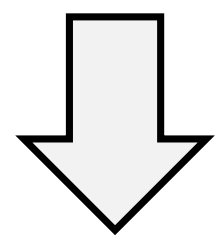

An interpolation method has been developed to increase artificially the number of SPST maps 


\section{SPST maps interpolation}

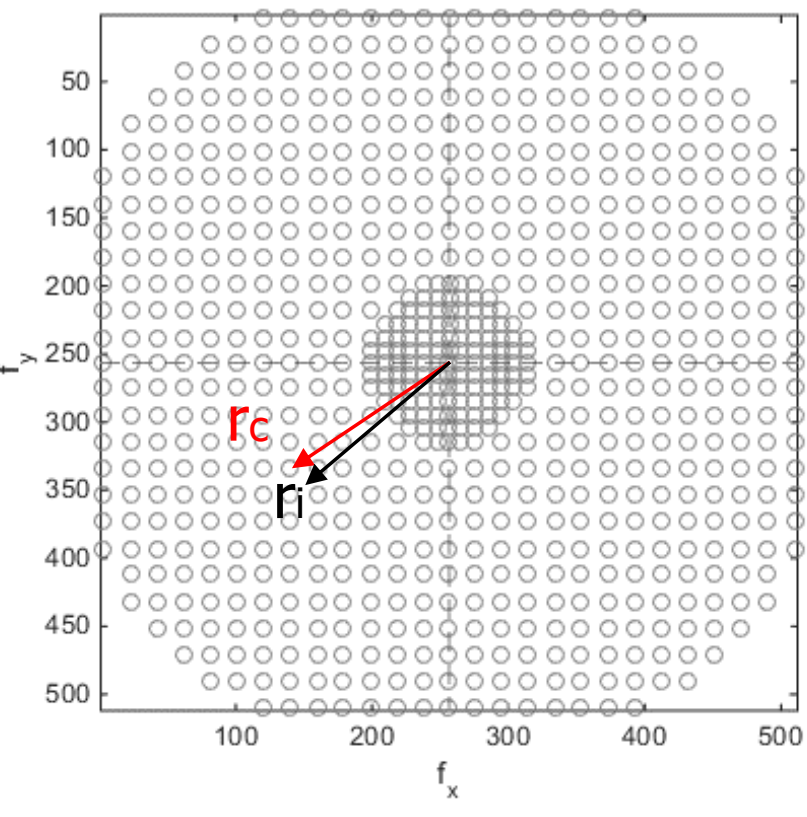

SPST map at a given pixel is obtained by performing a transformation (scaling and rotation) to the closest calibrated SPST maps

$$
s=\frac{\left|\boldsymbol{r}_{\boldsymbol{i}}\right|}{\left|\boldsymbol{r}_{\boldsymbol{c}}\right|} \quad \alpha=\cos ^{-1}\left(\left\langle\boldsymbol{r}_{\boldsymbol{i}} \mid \boldsymbol{r}_{\boldsymbol{c}}\right\rangle\right)
$$

- Several neighbors are used to fill the gaps at the edge

- Close to center: take closest neighbor with no scaling (denser calibration grid)
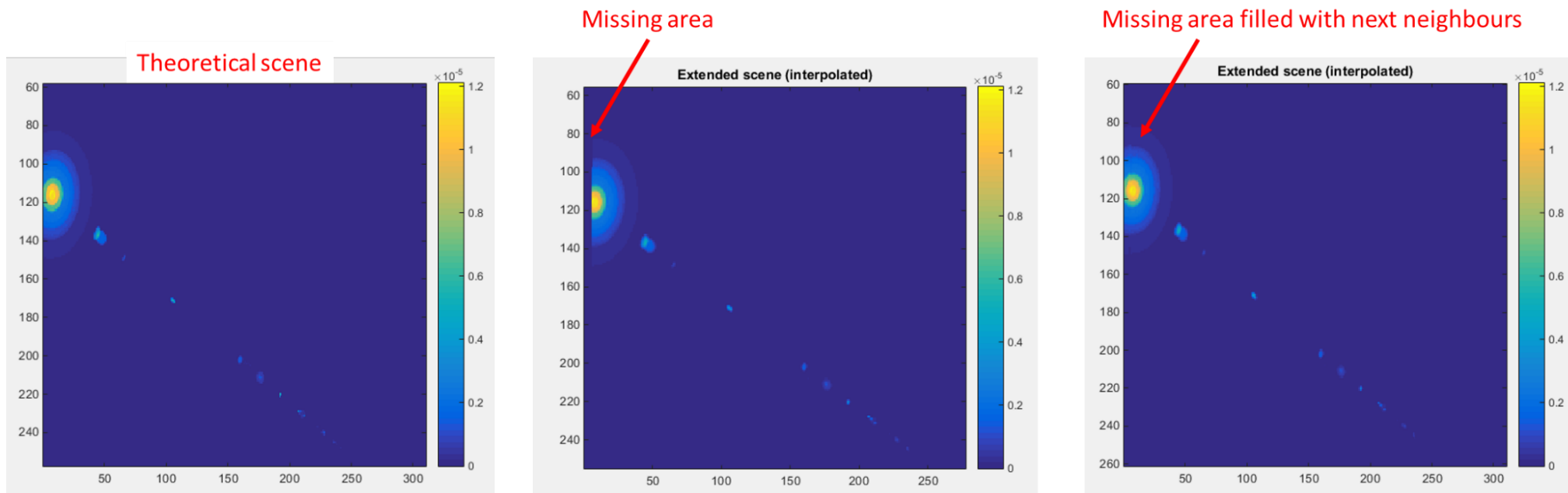


\section{With the interpolation method, the correction performance requirement is achieved}
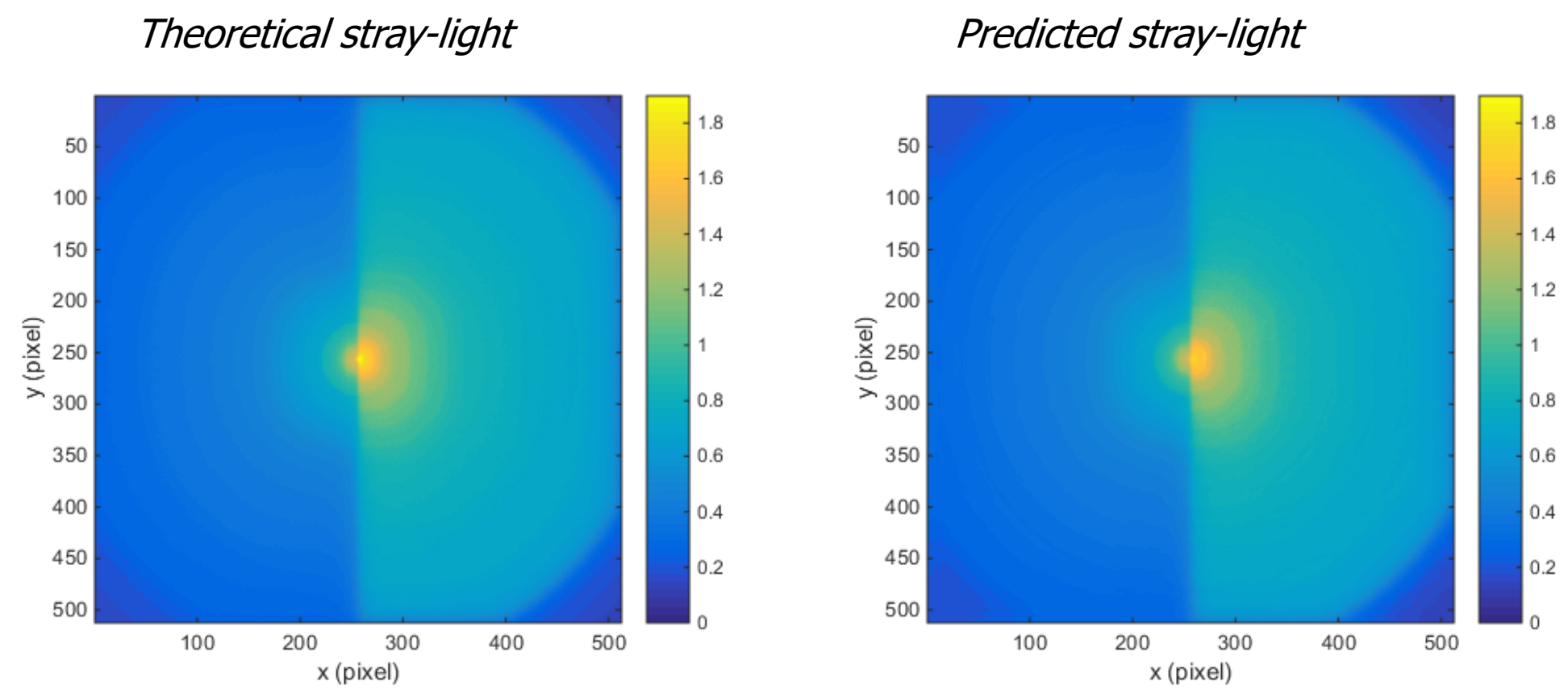


\section{With the interpolation method, the correction performance requirement is achieved}

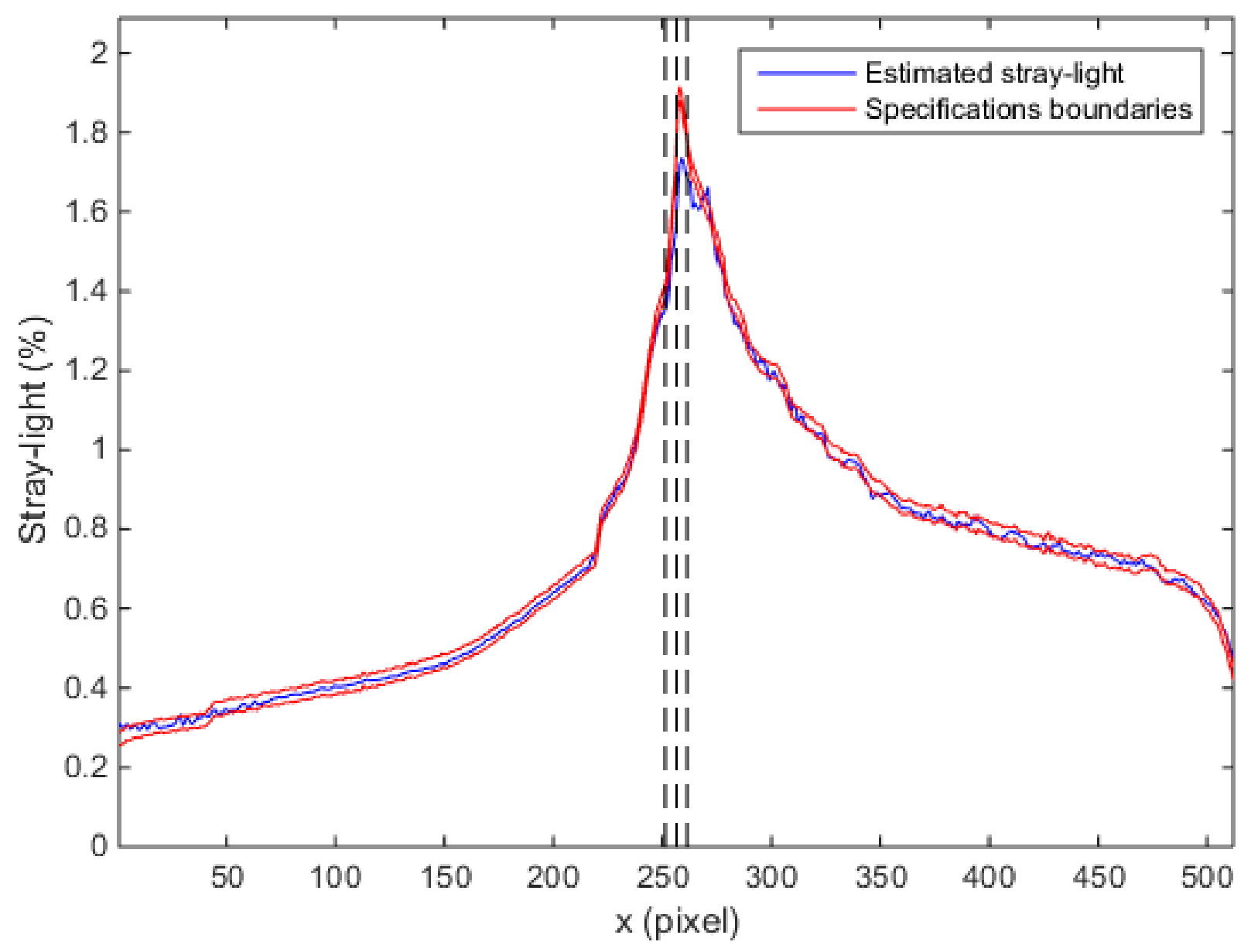

Residual stray-light: 0.148\% @2sigma 


\section{SPST acquistions}

Dynamic range decomposition is necessary to distinguish low orders of magnitude features

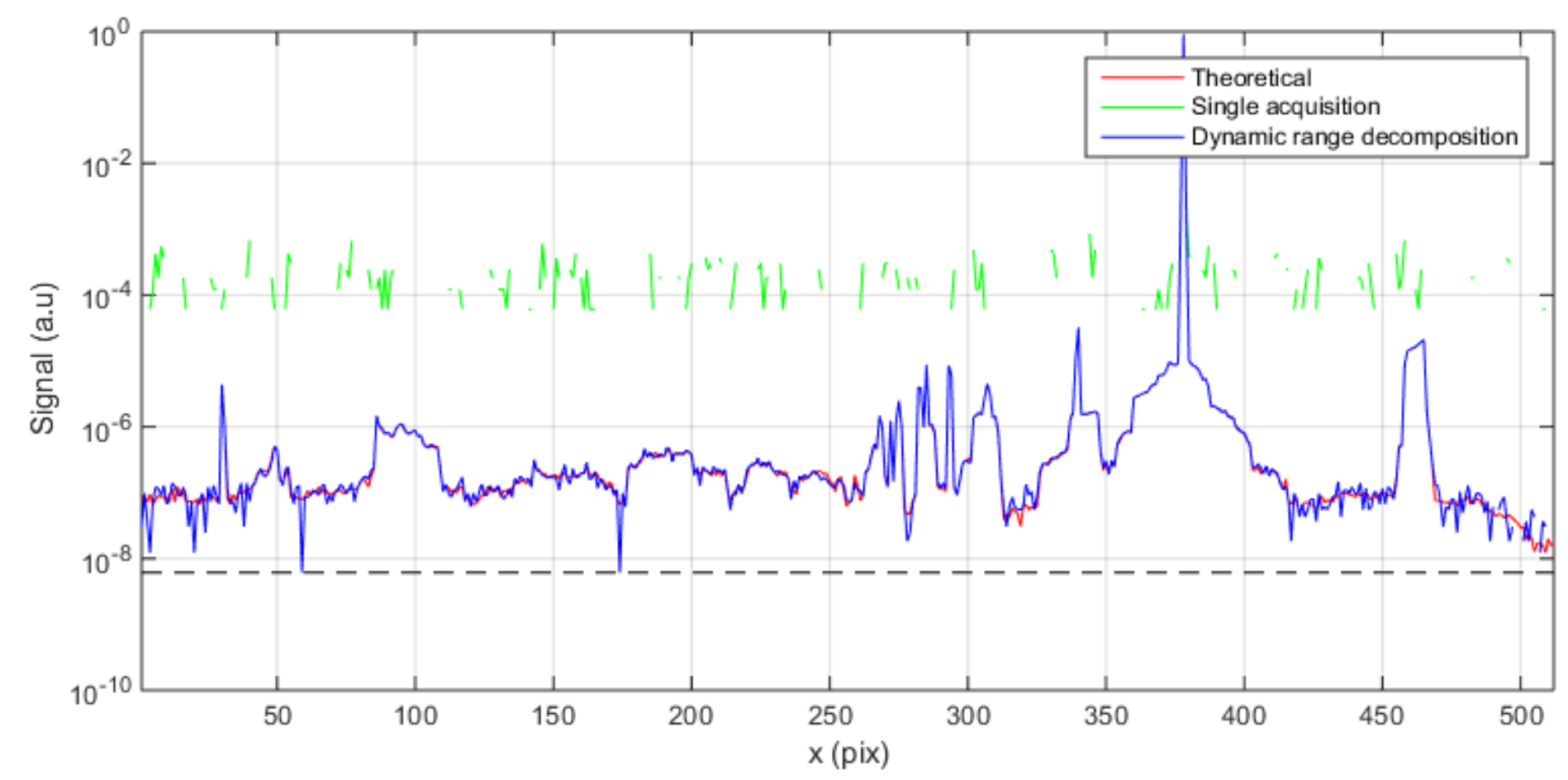

Considering the detector noise, the residual stray-light is $0.156 \%$ @2sigma 


\section{Conclusions}

\section{MI SL post-processing}

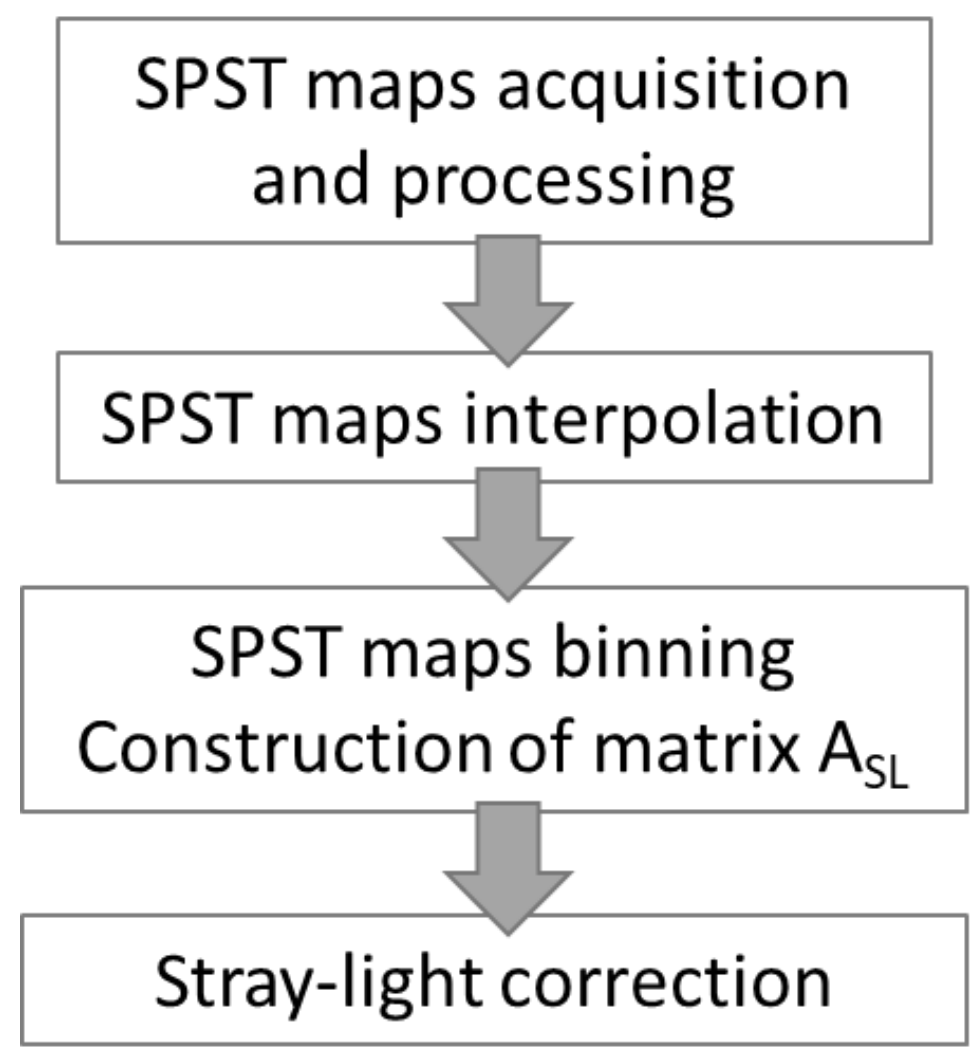

FoV grid selection $(27 * 27)$
Detector dynamic decomposition

Up to a grid of $256 * 256$

Up to a grid of $174 * 174$

2 iterations

Performances: SL level reduction by a factor 100 , reaching requirement 